\title{
Quo vadis? \\ Entwicklungstendenzen des deutschen Krankenhausmarktes
}

Deutschland hat nach den USA und der Schweiz das dritteuerste Gesundheitswesen der Welt. Jährlich
werden mehr als 10\% des Bruttoinlandproduktes (2004 ca. 234 Milliarden Euro) für die Gesundheit der
Bevölkerung aufgewandt. Allein 26\% (60,4 Mill. Euro) entfallen davon auf die Krankenhausversorgung [1].

Miriam E. S. Jung

Die Gesundheitsausgaben in Deutschland steigen seit 1995 pro Jahr um durchschnittlich 3,35\%. Damit wachsen sie stärker als das Bruttoinlandsprodukt. Diese Entwicklung wird durch eine Reihe von Faktoren beeinflusst. Die zunehmende Alterung der Bevölkerung, die mit einer erhöhten Morbidität einhergeht, führt zu einer erhöhten Inanspruchnahme von Leistungen bzw. steigenden Fallzahlen im stationären Sektor (1990 rund 14,3 Mio. Fälle und im Jahre 2004 16,8 Mio. Fälle) [2].
Durch den technischen Fortschritt, der nicht nur bessere, sondern meist auch teurere Behandlungsmethoden ermöglicht, wird diese Tendenz noch verstärkt. Die deutsche Krankenhausbedarfsplanung, die über Jahre hinweg zu Überkapazitäten an Krankenhausbetten geführt hat, trug zusätzlich zur Verschärfung der Gesamtssituation bei.

Um die Finanzierung der deutschen Gesundheitsversorgung auch zukünftig gewährleisten zu können, werden seit Jahren von der Politik Veränderungen im deutschen Gesundheitswesen mit dem Ziel der Kostendämpfung vorgenommen. Im Krankenhausbereich sollte eine Kostensteuerung durch Neuerungen in den Vergütungssystemen erreicht werden.

Seit Mitte der siebziger Jahre löste ein Vergütungssystem ein anderes ab. Keinem dieser Vergütungssysteme gelang es gleichzeitig Anreize zu schaffen, sowohl das kostengünstigste Behandlungsschema zu wählen als auch dieses möglichst kosteneffektiv zu erbringen.

Erst die seit 2004 eingeführte Fallpauschalenvergütung auf Basis der Diagnosis Related Groups (DRGs) konnte dies erreichen. Durch die DRGs erhält zukünftig jede Klinik für die gleiche erbrachte Leistung den gleichen Erlös, wodurch die Kliniken zur Verbesserung der Effizienz und Wirtschaftlichkeit gezwungen werden. Um zu verhindern, dass die nötigen Kosteneinsparungen zu Qualitätseinbußen führen, wurden parallel zu den Veränderungen in den Vergütungssystemen Qualitätssicherungsmaßnahmen zur Erhöhung der Transparenz eingeführt.

$\mathrm{Zu}$ den Herausforderungen durch die oben genannten Neuerungen im Krankenhausbereich kam erschwerend
Miriam E. S. Jung, Stabsbereich Unternehmensstrategie und Beteiligungscontrolling, BKK Bundesverband, Essen 
hinzu, dass es nicht gelungen ist, parallel zu den Umstellungen in der Vergütung auch die Finanzierung der Krankenhäuser grundlegend zu ändern.

In Deutschland erfolgt bis heute eine duale Krankenhausfinanzierung, das heißt, dass die laufenden Betriebskosten von den Krankenkassen und die Investitionen von den Bundesländern finanziert werden. Aufgrund leerer Länderkassen funktioniert dieses System nicht mehr ausreichend und verursacht einen Investitionsstau von schätzungsweise 25-50 Mrd. Euro [3]. Den Kliniken fehlen daher die finanziellen Mittel für die dringend notwenigen Sanierungen der überalterten Bausubstanzen und Neuanschaffungen von Gerätschaften.

Diese Ausgangssituation stellt die Kliniken vor große Herausforderungen. Der vom Gesetzgeber angestrebte Wettbewerb zwingt die Kliniken ihre bisherigen Arbeitsweisen grundlegend zu überdenken und zu ändern.

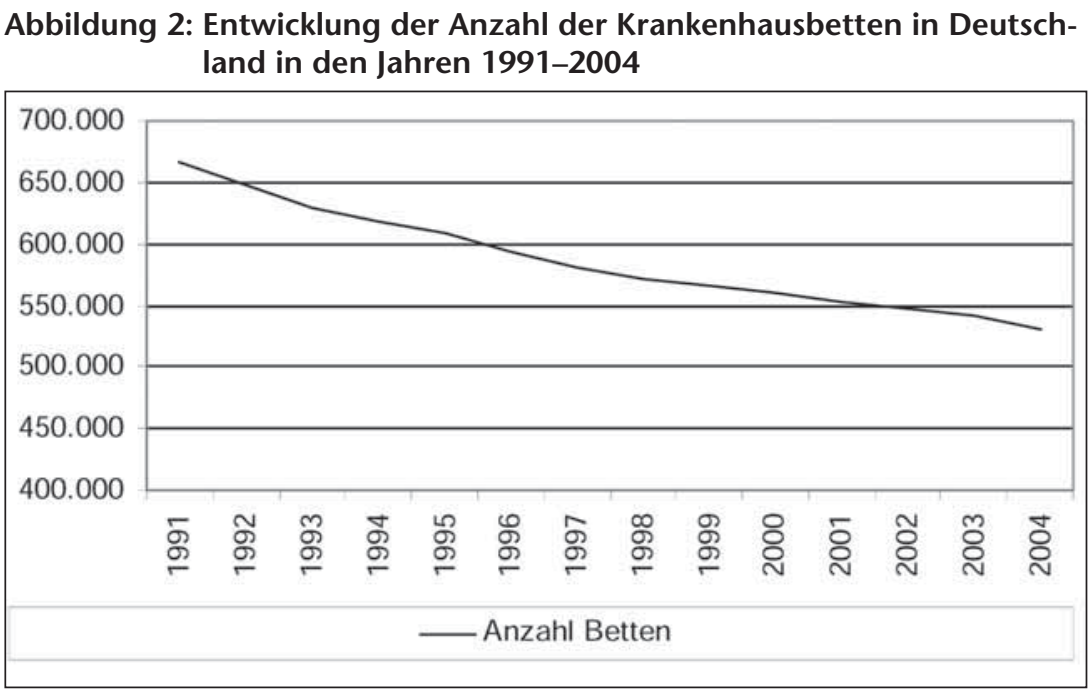

Quelle: Statistisches Bundesamt [5]

Abbildung 3: Entwicklung der Anzahl der Krankenhäuser in Deutschland nach Trägerschaft in den Jahren 1990-2005

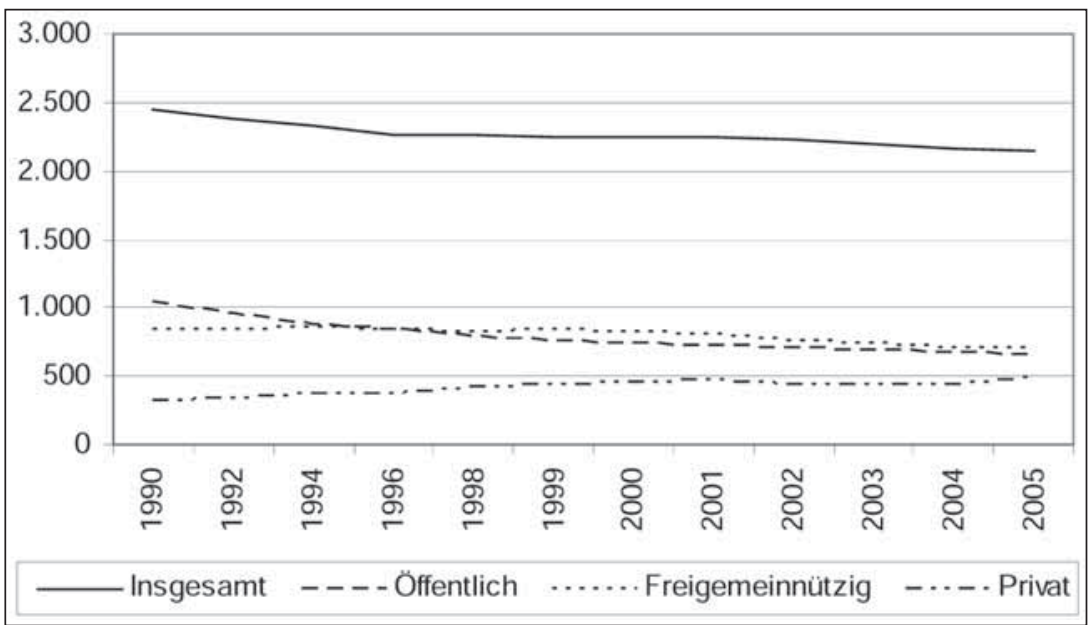

Quelle: Deutsche Krankenhausgesellschaft [2]
Die große Anzahl an Klinikschließungen in den letzten Jahren zeigt, dass viele Häuser den politisch gewollten Darwinistischen Kampf ("survival of the fittest") bereits verloren haben. Während es in Deutschland 1990 noch 2.447 Kliniken gab, bestanden fünfzehn Jahre später nur noch 2.139 Häuser [4]. Damit geht ein Rückgang der Krankenhausbetten einher [5].

Betrachtet man allein die Entwicklung in den Jahren 2004/2005, so zeigt sich, dass sich besonders die Anzahl von Häusern in öffentlicher Trägerschaft stark verringert hat. Sie verloren innerhalb eines Jahres 24 Krankenhäuser, davon überwiegend Häuser mit großen Bettenzahlen. Mit 712 Häusern konstant geblieben ist die Anzahl an freigemeinnützigen Häusern, allerdings bei Verringerung der Bettenanzahl um 2,10\% [5]. Lediglich die Anzahl der Häuser in privater Trägerschaft steigt kontinuierlich an. Allein im Zeitrahmen 2004/2005 erhöhte sich die ihre Anzahl von 444 auf 487 Häuser [4]. Trotz der bereits vorgenommen Schließungen scheinen weitere 20-30\% der aktuell existierenden Kliniken insolvenzgefährdet zu sein [6].

Um ihre Existenz zu sichern müssen die Kliniken drei Kriterien erfüllen:

1. Die Kliniken müssen ihre Leistungen zumindest kostendeckend erbringen.

2. Zur Tätigung notwendiger Investitionen müssen finanzielle Mittel beschafft werden.

3. Um sich auch langfristig im Wettbewerb behaupten zu können, muss die Marktmacht erhöht werden.

Um die gesetzten Ziele zu erreichen, verfolgen die Krankenhäuser eine Vielzahl unterschiedlicher Strategien, die teilweise in Abhängigkeitsbeziehungen zueinander stehen.

In den letzten Jahren drängen vermehrt private Krakenhausträger auf den deutschen Krankenhausmarkt. Diese gewinnorientiert arbeitenden Unternehmen sind zum Teil an der Börse notiert. Die deutsche Krankenhausbranche gilt in nationalen und internationalen Finanzinvestorenkreisen als besonders attraktiv, da die Erzielung einer hohen Rendite bei erfolgreicher Sanierung des Krankenhauses durchaus realistisch scheint [6]. Neuste Erhebungen bestätigen, dass ein privater Klinikbetreiber mit einer EBITDA-Marge von mindestens 3\% Prozent im ersten Jahr und bis zu 15\% und mehr im fünften Jahr nach Übernahme rechnen kann [7]. Da die privaten Krankenhausträger über die dringend benötigten finanziellen Investitionsmittel verfügen, wird ihnen der Markteintritt erheblich erleichtert. Auf diesem Wege können sie sich ein großes Stück des „Kuchens Kranken- 
Tabelle 1: Kennzahlen einiger privater Klinikketten in Deutschland im Jahre 2005

\begin{tabular}{|l|c|c|c|}
\hline & Umsatz in Mio. Euro ca. & Anzahl Betten ca. & Anzahl Mitarbeiter ca. \\
\hline $\begin{array}{l}\text { Asklepios Kliniken Verwaltungsgesellschaft mbH } \\
\text { (incl. Pacific Health Corporation) [9] }\end{array}$ & 1.900 & 20.000 \\
\hline Damp Holding AG [10] [11] & 357 & 4.100 \\
\hline Fresenius AG (incl. Helios) [12] & 2.009 & 26.000 \\
\hline KMG Kliniken AG [13] & 161 & 2.000 \\
\hline Marseille-Kliniken AG [14] [15] & 210 & 7.400 \\
\hline MediClin AG [16] [17] & 370 & 8.600 & 2.287 \\
\hline Paracelsus-Kliniken Deutschland GmbH [18] & 273 & 7.700 \\
\hline Rhön-Klinikum AG [19] & 1.416 & 4.230 \\
\hline Sana GmbH Co. KGaA [20] & 758 & 12.217 & 5.000 \\
\hline Schön Kliniken GmbH [21] & 301 & 6.740 \\
\hline SRH Kliniken GmbH [22] & 334 & 2.83 & 21.226 \\
\hline
\end{tabular}

Quelle: Eigene Darstellung

hausmarkt" mit einem Marktvolumen von rund 60 Milliarden Euro sichern [8].

$\mathrm{Zu}$ den bestimmenden Spielern im deutschen Krankenhausmarkt zählen heute vor allem private Klinikketten.

Bei den Ketten herrscht eine regelrechte Goldgräberstimmung; sie kaufen fast alles, was auf dem Markt angeboten wird. Als besonders beliebte Kaufobjekte gelten kleine bis mittelgroße Akutkliniken in ländlicher Monopollage. Durch die aggressiven Aquisitionsstrategien wird sich der geschätzte Anteil der privaten Akutkliniken in zehn Jahren auf bis zu 40\% erhöht haben [23] [24].

In letzter Zeit kommt auch der Kauf von ausgewählten Universitätskliniken in Mode, diese dienen aber eher als Prestigeobjekte als zur Gewinnerzielung. Neben diesen Häusern interessieren sich die privaten Anbieter für den Erwerb und den Aufbau von Spezialkliniken, die auf besonders „lukrative” Diagnosen fokussiert ("Rosinenpicken") sind. Aufgrund der bisher beobachteten Bestrebungen ist davon auszugehen, dass sich bis zum Jahre 2015 rund 40-45\% aller deutschen Krankenhausbetten in privater Trägerschaft befinden werden [23] [24].

Den anderen Häusern, z.B. Maximalversorgern und Universitätskliniken, die wegen geringer Attraktivität nur vereinzelt auf den Einkaufslisten der privaten Krankenhausträger stehen, bietet sich die Möglichkeit, die fehlenden finanziellen Mittel durch sogenannte „Public Private Partnership-Modelle" (PPP) zu erlangen. Unter einem PPP-Modell versteht man eine „langfristig vertraglich geregelte Zusammenarbeit zwischen öffentlicher Hand und Privatwirtschaft, bei der die erforderlichen Ressourcen (z.B. Knowhow, Betriebsmittel, Kapital, Personal etc.) von den Partnern zum gegenseitigen Nutzen in einem gemeinsamen Organisationszusammenhang eingestellt und vorhandene Projektrisiken entsprechend der Risikomanagementkompetenz der Projektpartner optimal verteilt werden [25]."

Grundsätzlich existiert in der Praxis eine Vielzahl von Gestaltungsmöglichkeiten der PPP-Verträge. So können PPP-Modelle sowohl für ein ganzes Klinikum als auch für Teilbereiche abgeschlossen werden. Der große Vorteil von PPP-Modellen liegt darin, dass die öffentliche Hand, im Gegensatz zu gewöhnlichen Privatisierungen, während der gesamten Vertragslaufzeit weiterhin Einfluss nehmen kann [26]. Da die Einführung von PPP-Modellen wegen hoch komplexer Vertragsstrukturen mit sehr hohen Transaktionskosten verbunden ist, amortisiert sich die Nutzung erst bei Projekten mit einem Volumen von 20-40 Mio. Euro [26]. Sowohl der Privatisierung als auch den PPP-Modellen wird nicht nur zugeschrieben, Lösungen für fehlende Investitionsmittel zu bieten, sondern auch die Krankenhäuser in ihrem Bestreben, die Leistungen zumindest kostendeckend zu erbringen, vorteilhaft zu beeinflussen. Den privaten Krankenhausträgern und den Unternehmen der PPP-Verträge wird zugesprochen, das dringend erforderliche betriebswirtschaftliche Know-how in die Krankenhäuser zu bringen. Dieses Wissen fehlt den meisten Kliniken auch heute noch, so dass viele Kliniken immer noch nicht in der Lage sind kostendeckend zu arbeiten.

Um dies zu ändern, sind Prozessoptimierungen dringend notwendig. Dabei besteht die Hauptaufgabe darin, die über Jahrzehnte gepflegten Ineffizienten zu beseitigen. Diese Fähigkeiten besitzen nach gängiger Meinung besonders die privaten Krankenhausbetreiber und die Vertragsunternehmen der PPP-Modelle. 
Allerdings darf nicht unerwähnt bleiben, dass die herausragenden positiven Ergebnisse privater Klinikanbieter nicht nur auf Prozessoptimierungen zurückzuführen sind. Die privaten Anbieter besitzen gegenüber den öffentlichen oder freigemeinnützigen Häusern weitere Vorteile, die sich maßgeblich auf den Unternehmenserfolg auswirken. So unterliegen die privaten Träger, bei Verzicht auf öffentliche Fördermittel, nicht mehr zwangsläufig dem öffentlichen Dienst-, Bau- und Einkaufsrecht [27]. Diese Sonderstellung ermöglicht es Personalkosten zu sparen, indem z.B. von den BAT-Tarifen des Öffentlichen Dienstes auf sogenannte Haustarife umgestellt wird. Bei einem Personalkostenanteil von über $60 \%$ kann dies eine große Kostenersparnis gegenüber der Konkurrenz bedeuten [7]. Durch die so gewonnen Freiheiten im Baurecht und die Finanzkraft der privaten Träger können vorhandene, bauliche Begebenheiten, die sich negativ auf die Wirtschaftlichkeit der Geschäftsprozesse (z.B. zu kleine Abteilungsgrößen) auswirken, schneller behoben werden.

Weitere notwendige Kosteneinsparungen können durch Nutzung von Synergie- und Skaleneffekten realisiert werden.

Den Kliniken stehen dazu die Möglichkeiten von Fusionen und Kooperationen zur Verfügung. Jedes fünfte deutsche Krankenhaus hat gemäß des „1. Berichtes zur Lage der Krankenhäuser in Deutschland bei Einführung der Fallpauschalen 2004" bereits die Möglichkeit einer Fusion geprüft, um sowohl auf Kosten- als auch auf der Markt/Nachfrageseite Verbesserungen zu erzielen. Fusionsbestrebungen sind sowohl bei Häusern in öffentlicher, freigemeinnütziger und privater Trägerschaft zu beobachten. Den Fusionsmöglichkeiten wurden durch das Bundeskartellrecht deutliche Grenzen gesetzt. Obwohl vielfach die Meinung vertreten wird, dass der Krankenhausmarkt aufgrund der hohen öffentlich-rechtlichen Regulierung kaum Spielräume für Wettbewerb lässt und der Missbrauch einer marktbeherrschenden Stellung aufgrund gesetzlich festgelegter Preise ausgeschlossen werden kann, daher die Anwendung des Kartellrechts keinen Sinn macht, entschied das Oberlandesgericht Düsseldorf, dass der deutsche Krankenhausmarkt der Fusionskontrolle durch das Bundeskartellamt unterliegen soll [27] [28]. Bis zum Jahre 2005 sind allein 50 Krankenhausfusionen, teilweise mit mehr als zwei Fusionspartnern, geprüft worden. Die Mehrzahl der Fusionen wurde genehmigt, lediglich zwei Fusionen wurden im März 2005 vollständig untersagt und eine Fusionserlaubnis erfolgte unter Auflagen. Bisher wurden lediglich nationale Krankenhauszusammenschlüsse beobachtet. Daher macht im Moment ein angestrebter Zusammenschluss des deutschen Universitätsklinikums Aachens mit dem niederländischen Universitätsklinikums Maastricht aufgrund der Überschreitung von Staatengrenzen Furore [29].

Im Gegensatz zu Fusionen geht bei einer Kooperation die rechtliche Eigenständigkeit der Krankenhäuser nicht verloren. Daher bevorzugen einige Kliniken diesen freiwilligen Zusammenschluss, der lediglich durch einen Vertrag geregelt ist. Während eine Kooperation meist von kurzer Dauer ist, bezeichnet man eine längerfristig angelegte Zusammenarbeit von Kliniken als Strategische Allianz. In der Praxis sind sowohl horizontale als auch vertikale Kooperationen zu beobachten. Unter einer horizontalen Kooperation wird in diesem Zusammenhang die Zusammenarbeit mehrerer Kliniken verstanden. Die oft als Klinikverbünde bzw. Kliniknetze bezeichneten Modelle bieten den Häusern die Möglichkeit, bestimmte Leistungen aus dem eigenen Portfolio in Abstimmung mit den Kooperationspartnern herauszunehmen oder bestimmte Leistungen zusammenzulegen. Letztere Variante wird im Hinblick auf die gesetzlich geforderten Mindestmengen bei bestimmten Krankheitsbildern (z.B. Brustkrebs) in Form von Zentrenbildung immer beliebter. Von einer vertikalen Kooperation wird gesprochen, wenn Krankenhäuser mit vor- oder nachgelagerten Leistungserbringern eng zusammenarbeiten. Diese Form der Kooperation wird meist in Form von Verträgen der integrierten Versorgung geschlossen. Krankenhäuser verfolgen durch die Zusammenarbeit mit vor- und nachgelagerten Leistungserbringern das Ziel ihre Steuerungsmöglichkeiten auf die Patientenströme ausbauen.

Vor diesem Hintergrund kommen auch Medizinische Versorgungszentren (MVZ) immer mehr in Mode. Das Konzept der Medizinischen Versorgungszentren orientiert sich am Modell der Polikliniken in der ehemaligen DDR. Medizinische Versorgungszentren sind fachübergreifende, ärztlich geleitete Einrichtungen, in der Ärzte sowohl als Inhaber (Vertragsärzte) als auch als Angestellte tätig sind. Die Gründung von Medizinischen Versorgungszentren wurde vom Gesetzgeber durch die Gesundheitsreform 2004 ermöglicht. Zum 31.12.2006 gab es in Deutschland bereits 666 Medizinische Versorgungszentren, mit stark steigender Tendenz [30]. Viele dieser Medizinischen Versorgungszentren befinden sich in der Trägerschaft von Krankenhäusern. Diese verfolgen durch Gründung von Medizinischen Versorgungszentren das Ziel, neue Patienten/Kunden für ihr Krankenhaus zu generieren und durch die Möglichkeit von preiswerteren und schneller durchgeführten Voruntersuchungen Kosten zu sparen. Ferner eröffnen die Medizinischen Versorgungszentren die Möglichkeit, die Fachgebiete des Krankenhauses abzurunden oder zu ergänzen [27].

\section{Fazit:}

Die deutschen Krankenhäuser stehen heute vor großen Herausforderungen. Um diese zu bewerkstelligen steht eine Vielzahl von Möglichkeiten zur Verfügung. Bei dem Bestreben Kosten durch Erzielung von Synergie- und Skaleneffekten zu senken, schneiden Kooperationen deutlich schlechter ab als Fusionen, da diese Verbindungen aufgrund ihrer Unverbindlichkeit eine geringere Halbwertzeit haben und durch die weiterhin bestehende rechtliche Selbstständigkeit jede Entscheidung nach wie vor für jedes Haus individuell getroffen wird [31]. Aber auch durch Fusionen erreichen viele Kliniken ihre angestrebten Ziele nicht. Als Gründe dafür sind hauptsächlich die fehlerhafte Auswahl von Fusionspartnern zu sehen, 
die sich in Bezug auf Stärken und Unternehmensleitbilder zu sehr unterscheiden [32]. Allein 70\% aller Fusionen von Krankenhäusern in öffentlicher oder kirchlicher Trägerschaft wurden als Misserfolge bezeichnet. Die Erfolglosigkeit kann durch fehlende Managementfähigkeiten und durch Entscheidungen, die mehr nach politischen als nach wirtschaftlichen Gesichtpunkten getroffenen worden sind, erklärt werden [32]. Obwohl die privaten Träger diese Probleme nicht handhaben müssen, werden immerhin 25\% der Fusionen von privaten Trägern als unbefriedigend beurteilt [32].

Die Mehrzahl von Beobachtern des deutschen Gesundheitsmarktes prophezeien eine starke Erhöhung der Anzahl der Häuser in privater Trägerschaft, so dass diese den Markt zukünftig dominieren werden. Dennoch haben nach Meinung der Autorin auch die Häuser in freigemeinnütziger Trägerschaft großes Potential den Markt zunehmend $\mathrm{zu}$ beeinflussen. Interessanterweise schneiden die privaten Häuser, entgegen verbreiteter Annahmen, z.B. hinsichtlich der durchschnittlichen Verweildauer deutlich schlechter als freigemeinnützige Häuser ab. Auch in der Arbeitsverdichtung sind nicht die privaten, sondern die freigemeinnützigen Häuser führend [33]. Trotz der deutlichen Verbesserungen der letzten Jahre schneidet das deutsche Gesundheitswesen im Vergleich zu den meisten europäischen Nachbarländern weiterhin schlecht ab. Das Effizienzpotential der Branche wird immer noch auf ca. 2,5-3,7 Mrd. Euro beziffert [34]. Welche Trägerform das derzeitige Wettrennen schließlich gewinnen wird, bleibt abzuwarten.

Fakt ist aber, dass die derzeitigen Entwicklungen auf dem Krankenhausmarkt dazu führen werden, dass die altbekannte Form des autarken Krankenhauses in $\mathrm{Zu}$ kunft nicht mehr existieren wird. Die Entwicklungen in den USA zeigen, dass die wohnortnahe Versorgung auch in einem deutlich stärker marktwirtschaftlich orientierten Krankenhausmarkt beibehalten werden kann [35]. Fraglich ist dennoch, ob in Deutschland die wohnortnahe Versorgung, wie wir sie kennen und schätzen auch weiterhin gewährleistet werden kann. Ob die Patienten trotz aller Bemühungen zu den Gewinnern oder den Verlieren dieser Entwicklung zählen werden, wird die Zeit zeigen. Daher bleibt nur abschließend zu fragen: Deutscher Krankenhausmarkt, quo vadis?

\section{Literaturverzeichnis:}

[1] Statisches Bundesamt Deutschland, http://www.destatis.de/themen/ d/thm_gesundheit.php, Zugriff am 10.04.2007

[2] Deutsche Krankenhausgesellschaft, Krankenhausfälle 1960-2004, http://www.dkgev.de/pdf/1392.pdf, Zugriff am 09.04.2007

[3] Frädrich, A., Duale Krankenhausfinanzierung am Ende? vom 16.04.2006, http://www.medizin.de/gesundheit/deutsch/2578.htm, Zugriff am 06.04.2007

[4] Deutsche Krankenhausgesellschaft, Anzahl Krankenhäuser 19602004, http://www.dkgev.de/pdf/1390.pdf, Zugriff am 06.04.2007

[5] Statistisches Bundesamt Deutschland, Fachserie 12, Reihe 6.1.1, https://wwwec.destatis.de/csp/shop/sfg/bpm.html.cms.cBroker.cls?c mspath=struktur,vollanzeige $. \operatorname{csp} \& C S P C H D x=0000000000000 \& I D=1$ 017782, Zugriff am 08.04.2007

[6] Wettke, J., Deutscher Krankenhausmarkt im europäischen/internationalen Umfeld, in: Klauber J., Robra B.-P., Schellschmidt, H.;
Krankenhausreport 2006 - Schwerpunkt: Krankenhausmarkt im Umbruch, Schattauer Verlag, 2006

[7] Albat A., Patzak, M., Der Krankenhausmarkt in Deutschland: Sind positive Margen Privatsache?, http://www.pwc.de/portal/pup/ut/p/ kcxml/04, Zugriff am 06.04.2007

[8] OECD, OECD Health Data 2003: a comparative analysis of 30 countries, OECD Publication service, 2003

[9] Asklepios Kliniken Verwaltungsgesellschaft mbH, http://www.asklepios.com/Pressezentrum/, Zugriff am 06.04.2007

[10] Damp Holding AG, http://www.damp.de/content/view/42/54/ lang.de/, Zugriff am 06.04.2007

[11] Ärztekammer Schleswig-Holstein, http://www.aeksh.de/shae/2006/ 200601/h061032a.html, Zugriff am 07.04.2007

[12] Fresenius AG, http://www.fresenius-proserve.de/gb2006d.pdf, Zugriff am 07.04.2007

[13] KMG Kliniken AG, http://www.kmg-kliniken-ag.com/unternehmen/wirtschaftsdaten.html, Zugriff am 07.04.2007

[14] Marseille-Kliniken AG, http://www.marseille-kliniken.de/, Zugriff am 06.04.2007

[15] Marseille-Kliniken AG, http://www.marseille-kliniken.de/ge/pdfs/ geschber/D-Jahresabschluss.pdf, Zugriff am 06.04.2007

[16] MediClin AG, http://www.mediclin.de/aktuell/pressearchiv/2006/ presse_060330.php, Zugriff am 07.04.2007

[17] ABC New Media AG, http://www.finanznachrichten.de/nachrichten2006-11/artikel-7285370.asp, Zugriff am 06.04.2007

[18] Paracelsus-Kliniken Deutschland GmbH, Jahresbericht 2005

[19] Rhön-Klinikum AG, http://rka.hlp.de/rka/investorrelations, Zugriff am 06.04.2007

[20] Sana GmbH \& Co. KGaA, http://www.sana.de/download/sana_gb_ 2005.pdf, Zugriff am 07.04.2007

[21] Schön Kliniken GmbH, http://www.schoen-kliniken.de/PDF_QUB_ JB/50377_Jahresbericht_schoen.pdf, Zugriff am 07.04.2007

[22] SRH Kliniken GmbH, http://www.srh.de/cps/rde/xchg/SID3F575FEA5D377901/srh_dir/hs.xsl/373_1407_DEU_HTML.html, Zugriff am 06.04.2007

[23] Meder, G., Nur wer den Sieg im Wettbewerb nicht scheut, wird ihn erreichen. Warum der Investitionsdruck zu neuen Wegen in der Krankenhausfinanzierung zwingt, in: Führen und Wirtschaften, 2002, Nr. 2, S. 182-185

[24] Schmidt, C., Möller, J., Gabbert, T., Engeler, F., Investoren im Krankenhausmarkt. in: Deutsche Medizinische Wochenzeitschrift, 2003, Nr. 128, S.1551-1556

[25] BMVBW (Hrsg.), Gutachten: PPP im öffentlichen Hochbau, Band II: Rechtliche Rahmenbedingungen, Teilband II: Vergaberecht, Steuerrecht, Recht der öffentliche Förderung, 2003

[26] Alfen, H. D., Buscher, F., Daube, D. , Weidemann, A., Public Private Partnership im Krankenhausbereich, in: Das Krankenhaus, 2005, Nr.12, S. 1083-1088

[27] Flintrop, J., Pföhler, W., Die Vollversorgung in der Fläche ist das Ziel, in: Deutsches Ärzteblatt, 09.01.2006, Nr. 103, S. A-11/B-9/C-9

[28] Stüwe, H., Ulf Böge: „Krankenhausmonopole müssen wir verhindern", in: Deutsches Ärzteblatt, 07.10.2005, Nr. 102, S. A-2671/B2257/C-2133

[29] Universitätsklinikum Aachen, http://www.ukaachen.de/go/show? $\mathrm{ID}=5183044 \&$ ALTNAVID $=5111120 \& \mathrm{DV}=0 \& \mathrm{COMP}$ page\&ALTN, Zugriff am 06.04.2007

[30] Kassenärztliche Bundesvereinigung, Pressemitteilung vom 12.03.2007, http://www.kbv.de/, Zugriff am 06.04.2007

[31] Richter, E., Kooperation oder Fusion? - Kliniken haben die Wahl, in: Ärzte Zeitung vom 08.04.2004, http://www.aerztezeitung.de/docs/ 2004/04/08/066a2301.asp, Zugriff am 06.04.2007

[32] Gemeinsame Studie des Centrums für Krankenhausmanagement (CKM) und der Wirtschaftsprüfungs-Gesellschaft APB 2002, http://www.uniprotokolle.de/nachrichten/id/2172/, Zugriff am 06.04.2007

[33] IFG Institut für Gesundheitsökonomik, http://ifg-muenchen.com/, Zugriff am 06.04.2007

[34] Klauber J., Robra B.-P., Schellschmidt,H.; Krankenhausreport 2006 Schwerpunkt: Krankenhausmarkt im Umbruch, Schattauer Verlag, 2006

[35] Eckhardt, M., Die Entwicklung des Krankenhausmarktes in den USA, in: Klauber J., Robra B.-P., Schellschmidt, H.; Krankenhausreport 2006 - Schwerpunkt: Krankenhausmarkt im Umbruch, Schattauer Verlag, 2006 